\title{
Impact of damping uncertainty on SEA model response variance
}

\author{
Noah Schiller ${ }^{a}$ \\ Randolph Cabell ${ }^{\text {b) }}$ \\ Structural Acoustics Branch \\ NASA Langley Research Center \\ Hampton, VA 23681 USA \\ Ferdinand Grosveld ${ }^{\text {c) }}$ \\ Lockheed Martin \\ NASA Langley Research Center \\ Hampton, VA 23681 USA
}

\begin{abstract}
Statistical Energy Analysis (SEA) is commonly used to predict high-frequency vibroacoustic levels. This statistical approach provides the mean response over an ensemble of random subsystems that share the same gross system properties such as density, size, and damping. Recently, techniques have been developed to predict the ensemble variance as well as the mean response. However these techniques do not account for uncertainties in the system properties. In the present paper uncertainty in the damping loss factor is propagated through SEA to obtain more realistic prediction bounds that account for both ensemble and damping variance. The analysis is performed on a floor-equipped cylindrical test article that resembles an aircraft fuselage. Realistic bounds on the damping loss factor are determined from measurements acquired on the sidewall of the test article. The analysis demonstrates that uncertainties in damping have the potential to significantly impact the mean and variance of the predicted response.
\end{abstract}

\section{INTRODUCTION}

Statistical Energy Analysis (SEA) is often used to predict the high-frequency vibroacoustic response of aerospace vehicles. This energy based method requires significantly fewer degrees of freedom than conventional displacement based approaches (e.g. FEA); however predictions are only available in terms of mean response levels. Mean response levels can be computed over various populations, including averages over a population of similar structures, spatial averages over a subsystem, and frequency averages within a frequency band. While it is useful to know the mean response, in some cases it is important to know bounds on the response. For instance, the mean response is rarely used for design since actual response levels can deviate significantly from the mean. Therefore when design loads or test criteria are needed, additional factors are commonly added to the mean SEA value to account for ensemble, point-to-point, frequency,

\footnotetext{
a) Email address: noah.h.schiller@nasa.gov

b) Email address: randolph.h.cabell@nasa.gov

c) Email address: f.grosveld@nasa.gov
} 
and/or structural parameter variations. These factors are often based on experience or empirical databases ${ }^{1}$.

Instead of adding margins to the output, there has been significant interest in calculating response bounds within the framework of SEA. Much of this work has focused on calculating the ensemble variance of built-up systems ${ }^{2,3}$. Recall that SEA predicts the mean response over a population of similar structures, such as the mean response within similar vehicles leaving an assembly line. Therefore measurements acquired on a particular test structure will not necessarily match predictions. Ensemble variance quantifies the expected variability within a population of similar structures.

Lyon and DeJong ${ }^{2}$ presented one of the first comprehensive formulations for the ensemble variance of built-up systems. While instructive, subsequent work has shown that an underlying assumption regarding the statistical distribution of resonance frequencies is not appropriate for many systems ${ }^{4,5}$. Langley and Cotoni ${ }^{3}$ have since updated and expanded the ensemble variance derivation. Their analysis begins with the traditional SEA equations, however the coefficients in the equation are taken to be random and a perturbation expansion is used to determine the variance of the subsystem energies. VA One ${ }^{6}$ software has incorporated /DQJOH $\backslash$ DQG $\square \&$ RWRQLTV $\square$ formulation for ensemble variance. Therefore confidence intervals, accounting for ensemble variation, can now be predicted with commercially available software.

While ensemble variance is valuable, it accounts for just one source of uncertainty. Variations in input parameters, such as damping, are not included within the ensemble variance formulation. Although Langley and Cotoni outlined a procedure for propagating damping uncertainty through an SEA model, the importance of such an approach was not explicitly demonstrated $^{2,7}$. The present paper shows that damping loss factor uncertainty can significantly impact both the mean and variance of the predicted response. The analysis is performed on a stiffened, floor-equipped, composite cylinder that resembles an aircraft fuselage. The paper begins with a brief description of the test structure, which was recently tested at NASA Langley ${ }^{8}$. The measurement procedure used to estimate the damping loss factor from experimental data is then described. The next section describes the procedure for combining ensemble values, as calculated by VA One, with loss factor uncertainty. Finally an example is provided that demonstrates the importance of propagating loss factor uncertainty through SEA.

\section{LOSS FACTOR UNCERTAINTY}

\subsection{Test Structure}

Loss factor estimates were acquired on the sidewall of a floor-equipped composite cylinder. The test structure, shown in Fig. 1 , is $1.676 \mathrm{~m}$ in diameter and $3.658 \mathrm{~m}$ long. The $0.00170 \mathrm{~m}$ thick shell, which is made of carbon fiber filaments embedded in an epoxy resin, is reinforced by orthogonal supports (i.e. ring frames and stringers) that are riveted and bonded to the skin. These stiffeners segment the cylinder into 198 rectangular panels with nominal dimensions of $0.203 \mathrm{~m}$ by $0.381 \mathrm{~m}$. A $0.0127 \mathrm{~m}$ thick plywood floor is installed $0.544 \mathrm{~m}$ above the bottom of the cylinder and the entire structure is supported by two $0.0889 \mathrm{~m}$ thick particle board end caps. More details on this structure can be found in Jackson'. 

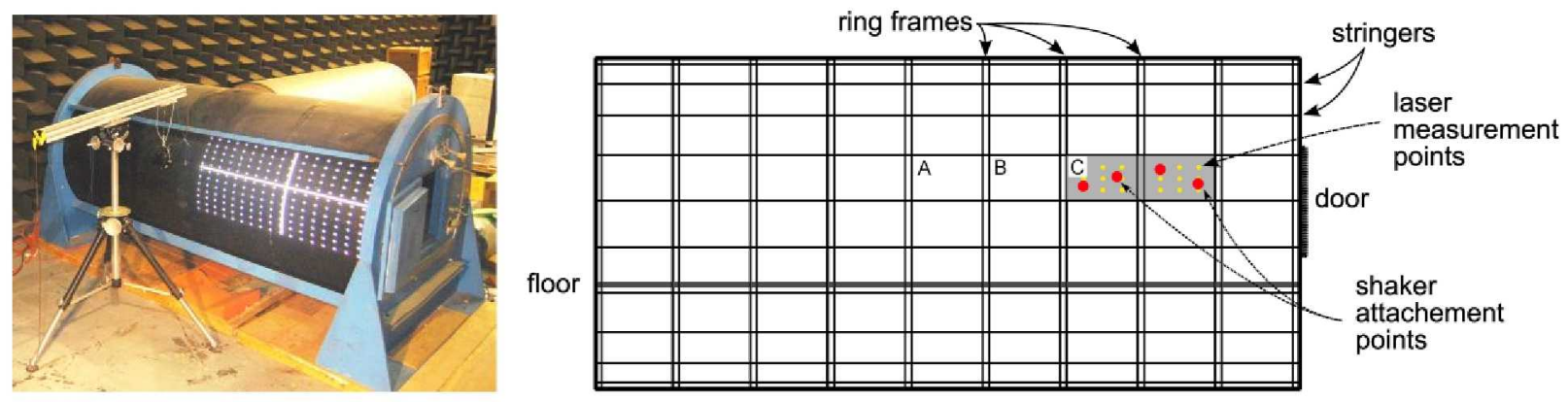

Fig. 1 - Photograph (left) and sketch (right) of the stiffened composite cylinder.

\subsection{Impulse response decay method (IRDM)}

Total loss factor estimates for the sidewall were acquired using the impulse response decay method (IRDM) $)^{10,11}$. This method is used to estimate damping from frequency response functions (FRFs). In this case, thirty-six unique FRFs were acquired with drive and measurement points spread over two different panels, as shown in Fig. 1. Specifically the frequency responses were between a force gage, attached to a shaker on the interior of the sidewall, and laser vibrometer measurements acquired on the outside of the cylinder.

Figure 2 illustrates the steps involved in IRDM. Each frequency response function (shown with blue in the figure on the left) is windowed to obtain the band-limited response (shown by the red curve). The window is shaped to match the magnitude response of a $4^{\text {th }}$-order Butterworth band-pass filter with cutoff frequencies aligned with the edges of the $1 / 3$-octave band of interest (shown with the dashed lines). The inverse Fourier transform is then used to compute the impulse response (shown with the blue line in the center figure) from each $1 / 3$ octave band windowed-FRF. The normalized Schroeder decay curve (red curve on the right) is then calculated from the impulse response ${ }^{12}$. A linear regression is used to determine the parameters of a simple exponential model that best fit the decay curve

$$
\ddot{\mathscr{Q}}(t)=c_{1} e^{-2 \pi f \eta t} \text {, }
$$

where $\ddot{d}$ is the estimate of the decay curve, $\eta$ is the loss factor, and $f$ is the center frequency of the band $d^{10}$. The square of the correlation coefficient, $R^{2}$, is then computed for each regression to quantify the correlation between the original curve and the fit. In this case a perfect fit is indicated by $R^{2}=1$. Since most curves are more complicated than a simple exponential decay, the results of the curve-fit are sensitive to the fitted portion of the decay curve. Therefore a constrained optimization is used in each $1 / 3$-octave band to select the fit range that gives the highest $R^{2}$ value. During the optimization, the fit range is constrained between -1 and $-20 \mathrm{~dB}$ of decay. Once the optimal fit range is determined and the fit is performed, screening is used to eliminate frequency responses with an $R^{2}$ value of less 0.98 . The purpose of this step is to eliminate bad estimates, which occur when the FRF does not contain sufficient dynamic response within the $1 / 3$-octave band ${ }^{10}$. 

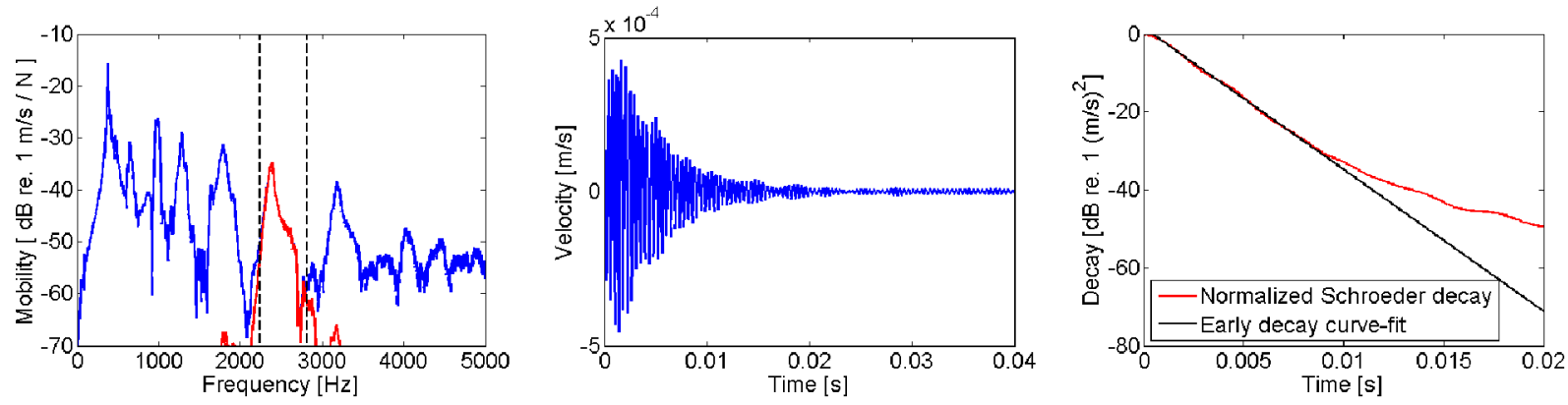

Fig. 2 - Figures highlighting the steps involved in IRDM. The figure on the left shows the $R U L J L Q D O \square D Q G \square I L O W H U H G \square) 5$ ) $V \square \square Z K L F K \square D U H \square X V H G \square W R \square J H Q H U D W H \square W K H \square 1$ the center figure. The figure on the right shows the Schroeder decay curve and the curve-fit.

The mean and variance of the total loss factor were then calculated from all IRDM estimates within each 1/3-octave band from 100 to $4000 \mathrm{~Hz}$. However to calculate confidence intervals it is necessary to know the shape of the distribution as well as the mean and variance. Therefore histograms of the loss factor estimates were used to estimate the shape of the distribution. Figure 3 compares two histograms with normalized log-normal probability density functions,

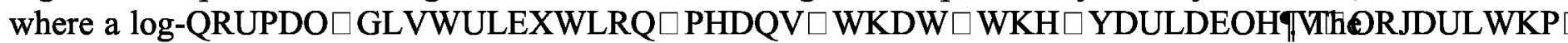
probability density function (PDF) for a log-normal distribution can be expressed as

$$
f_{X}(x ; \mu, \sigma)=\frac{1}{x \sigma \sqrt{2 \pi}} e^{-\frac{(\ln x-\mu)^{2}}{2 \sigma^{2}}}
$$

where $\mu$ and $\sigma$ are the mean and standard deviation of the variable's natural logarithm. Although the number of samples in the plots is too small to definitively characterize the distribution of the estimates, it is clear that the data is right-skewed and a log-normal distribution appears to be appropriate.
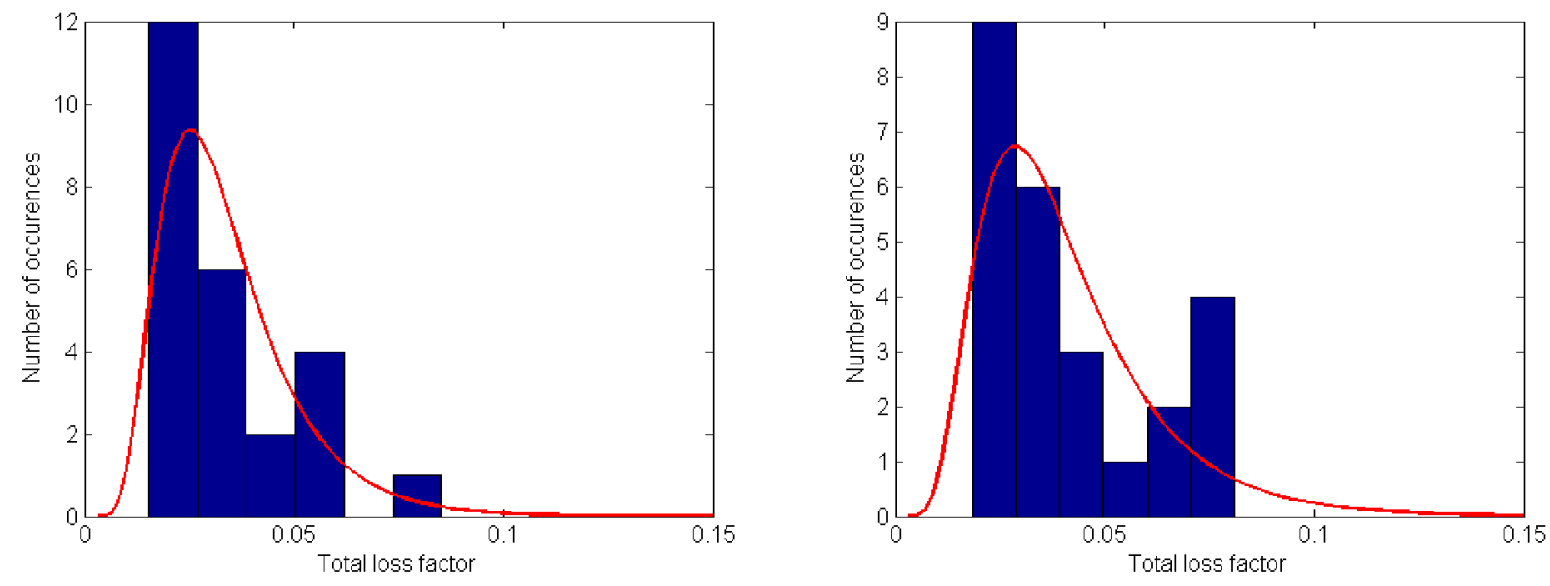

Fig. 3 - Histogram of the total loss factor estimates along with normalized log-normal PDFs in the $2500 \mathrm{~Hz}$ (left) and $3150 \mathrm{~Hz}$ (right) 1/3-octave bands.

The average loss factor for each 1/3-octave band is shown by the solid blue line in Fig. 4, along with the $90 \%$ confidence intervals indicated by the dashed lines. Notice that while the mean loss factor approaches 0.04 at high frequencies, the $90 \%$ confidence interval extends from 0.015 to 0.083 . 


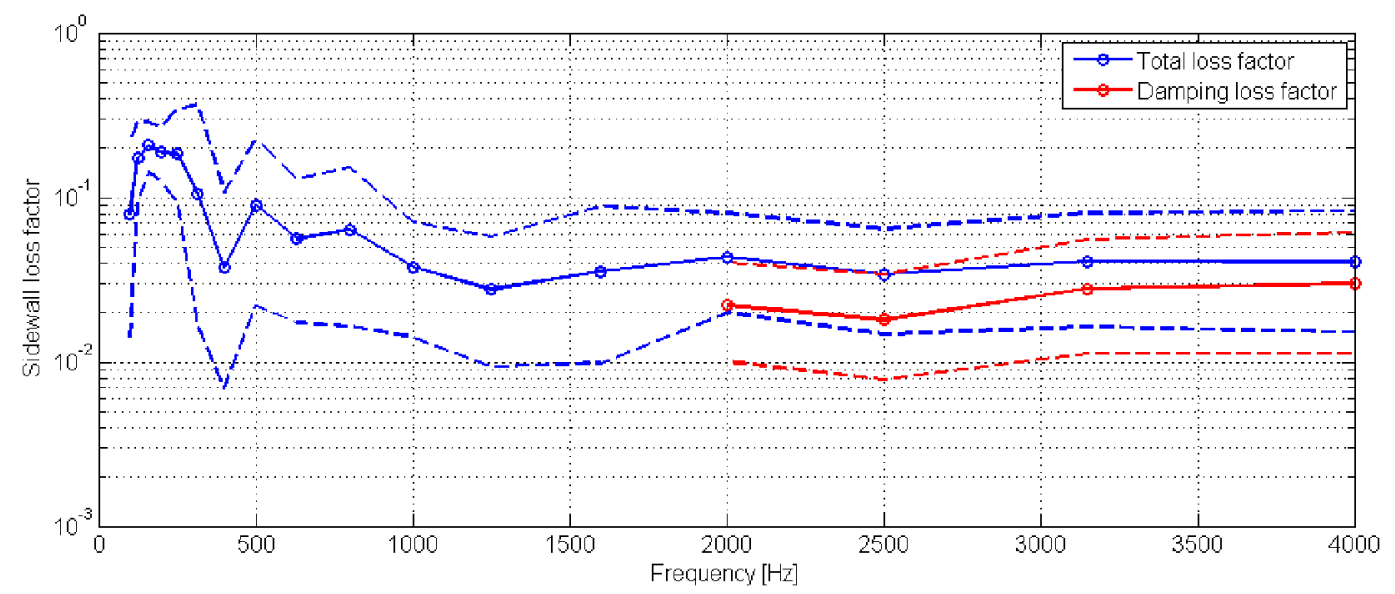

Fig. 4 - The blue curves show the measured total loss factor for the sidewall and the red curves show the estimated damping loss factor. The solid lines show the mean values and the dashed lines show the $90 \%$ confidence intervals.

The total loss factor estimated by the IRDM includes all energy loss mechanisms, including damping and transmission to other components. For example a system composed of two identical, coupled subsystems with damping $\eta$ and coupling $\eta_{12}$ will have a total loss factor of

$$
\eta_{T}=\eta+\frac{\eta \eta_{12}}{\eta+\eta_{12}}
$$

This simple example demonstrates that the total loss factor can be significantly larger than the damping loss factor if coupling is strong. For complex systems the relationship between the total loss factor and damping loss factor is not as simple as Eq. 3. Therefore an SEA analysis was used to estimate the damping loss factor from the total loss factor. An SEA model of the cylinder was created as described in Appendix A. The damping loss factor was varied within the model while estimating the total loss factor as $\eta_{T}=P / \omega E$, where $P$ is the input power and $E$ is the total energy in the driven subsystem. When the estimate of the total loss factor matched the measured value, then the damping loss factor was assumed to be correct. This approach assumes that the model is accurate. Since the accuracy of SEA tends to deteriorate at low frequencies, predictions below $2000 \mathrm{~Hz}$ were not used. While the mean values were updated using the SEA model, the variance was not. Instead the variance of the damping loss factor was assumed to equal the variance of the measured total loss factor. While this approach may not be rigorous, it provides a practical way to estimate damping values from total loss factor measurements. Damping loss factors are shown with red in Fig. 4.

The statistics of the damping loss factor can be visualized using the probability density function. As an example, Fig. 5 shows the PDF for the damping loss factor in the $2500 \mathrm{~Hz} \mathrm{1/3-}$ octave band. The circle depicts the mean value while the dashed lines indicate the $90 \%$ confidence interval. Notice that since the distribution is log-normal, the bounds are not symmetric about the mean. 


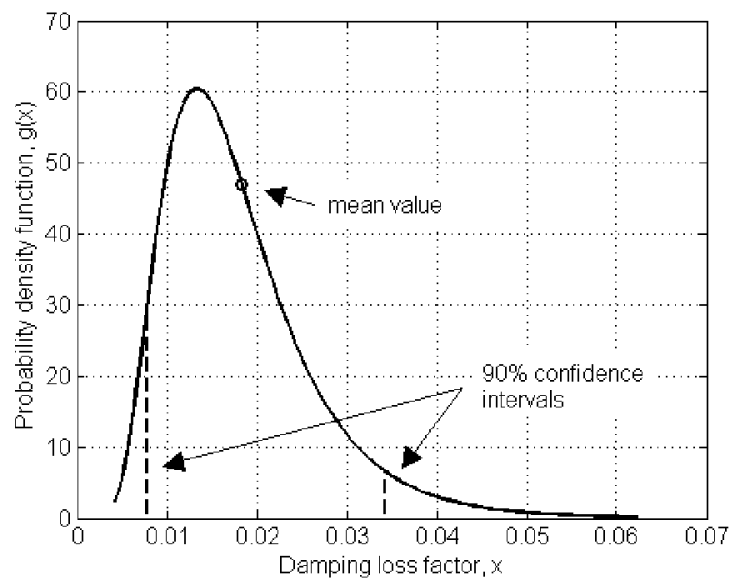

Fig. 5 - Probability density function for the damping loss factor in the $2500 \mathrm{~Hz}$ 1/3-octave band.

\section{COMBINED RESPONSE DUE TO ENSEMBLE VARIATION AND LOSS FACTOR UNCERTAINTY}

Statistical variation in the loss factor can be combined with ensemble variation using

$$
h(y)=\int_{-\infty}^{\infty} f(y \mid x) g(x) d x,
$$

where $g(x)$ is the probability density function for the damping loss factor, and $f(y \mid x)$ is the conditional density of the response $e^{13}$. The probability density function for the damping loss factor $g(x)$ can be determined from measured data as described in the previous section, and the conditional density of the response $f(y \mid x)$ can be determined using software such as VA One. Although VA One provides confidence intervals that account for ensemble variation, the conditional density function, $f(y \mid x)$, is not directly available. As stated in the software documentation $^{6}$, the statistical distribution of the subsystem energy is assumed to be log-normal. Therefore the confidence intervals are defined as

$$
E \in\left(e^{\mu-\sigma y^{*}}, e^{\mu+\sigma y^{*}}\right),
$$

where $E$ is the response variable (i.e. subsystem energy), $\mu \mathrm{LV} \square \mathrm{WKH} \square \mathrm{PHDQ} \square \mathrm{RI} \square \mathrm{WKH} \square \mathrm{YDULDEOH}\rceil \mathrm{V}$ logarithm, $\sigma$ is the standard deviation of the variable's natural logarithm, and $q^{*}$ is the $(1-\alpha / 2)$-quantile of the standard normal distribution. For $90 \%$ confidence intervals, $\alpha$ is 0.1 and $q^{*}=1.64$. Therefore once the confidence intervals are determined, $\mu$ and $\sigma$ can be found using Eq. 5 and then the conditional density function $f(y \mid x)$ can be determined with Eq. 2 . used,

If there are several uncertain system properties, the multivariable extension to Eq. 1 can be

$$
h(y)=\int_{-\infty}^{\infty} \int_{-\infty}^{\infty} \ldots \int_{-\infty}^{\infty} f\left(y \mid x_{1}, x_{2}, \ldots, x_{n}\right) g_{1}\left(x_{1}\right) g_{2}\left(x_{2}\right) \ldots g_{n}\left(x_{n}\right) d x_{1} d x_{2} \ldots d x_{n},
$$

where $f\left(y \mid x_{1}, x_{2}, \ldots, x_{n}\right)$ is the joint conditional distribution of $Y$ given that $X_{1}=x_{1}, X_{2}=x_{2}$, and $X_{n}=x_{n}$; and $g_{n}\left(x_{n}\right)$ is the PDF for the uncertain input parameter. By writing the expression in this form, it is assumed that $X_{1}, X_{2}$, through $X_{n}$ are all mutually statistically independent ${ }^{13}$. Unfortunately this approach becomes computationally expensive as the number of uncertain input parameters increases. This is because it is necessary to resolve the SEA equations and recalculate $f\left(y \mid x_{1}, x_{2} \square \ll \square \emptyset\right.$ for every unique combination of $\left(x_{1}, x_{2}, \ldots, x_{n}\right)$. Therefore in some situations it may 
be helpful to perform an initial sensitivity study to down select to the system properties that have the largest effect on the response before proceeding with the analysis. In this paper, the sidewall damping loss factor is the only uncertain parameter, so the simple expression given in Eq. 4 can be used for the analysis.

\section{RESULTS}

In order to illustrate the impact of damping uncertainty on response level prediction, the SEA cylinder model was used to predict panel responses due to a structural power input on the sidewall. Specifically the input was applied to panel A, identified in Fig. 9, and responses were observed on panels B and C. Panels B and C are located immediately to the right of the driven panel as shown in Fig. 9.

Initially damping uncertainty was neglected and VA One was used to calculate the mean response, along with $90 \%$ confidence intervals accounting for ensemble variation. In this case the mean damping loss factor, shown by the solid red line in Fig. 4, was used for all panels on the sidewall. The black curves in Fig. 6 show the mean response (solid line) along with the $90 \%$ confidence intervals (dashed lines) for panels B and C. The spread in the results is due to ensemble variation.
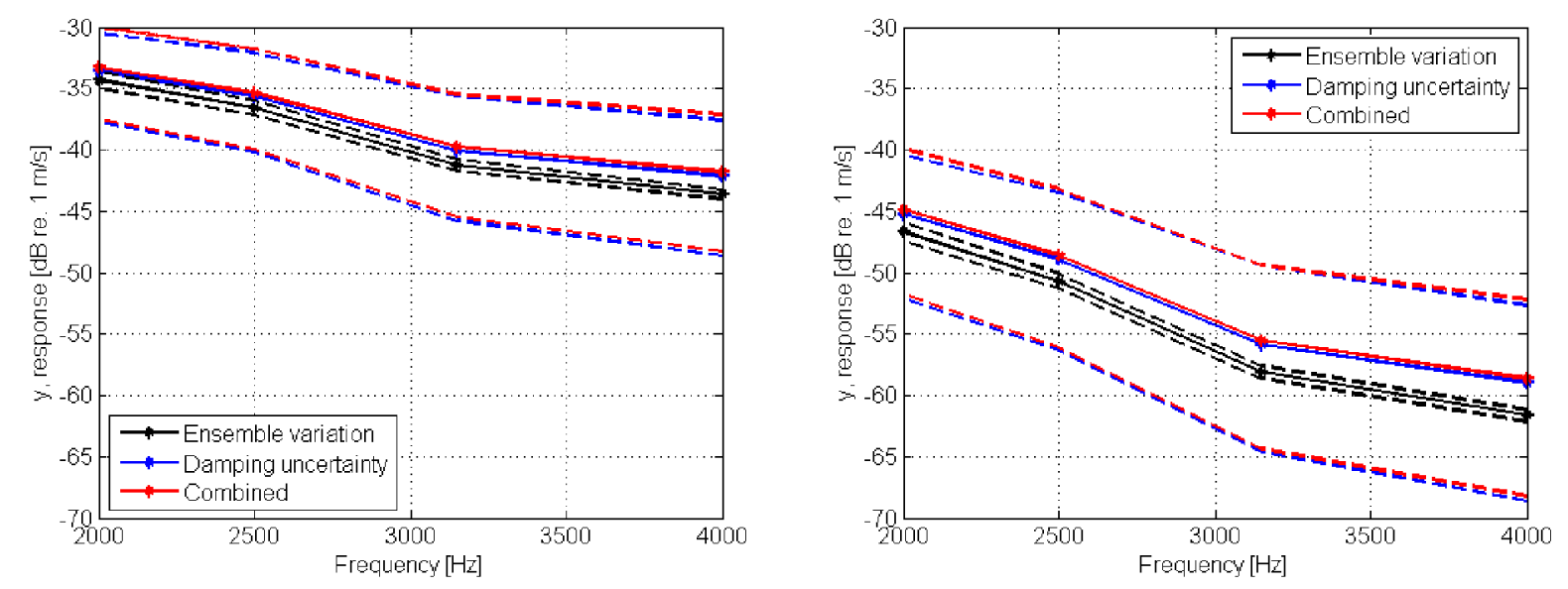

Fig. 6 - Predicted velocity on panel B (left) and panel $C$ (right). The black curves show the output of VA One, which accounts for ensemble variation, the blue curves account for damping uncertainty, and the red curves account for both damping uncertainty and ensemble variation. The solid lines designate the mean and the dashed lines show the 90\% confidence intervals. (The red curves have been shifted up by $1 / 3 \mathrm{~dB}$ for clarity.)

Next the impact of damping uncertainty was evaluated without incorporating ensemble variation. This was accomplished by solving the SEA equations multiple times while varying the sidewall damping loss factor. Again it was assumed that every panel on the sidewall had the same damping loss factor and therefore the damping of all panels was changed together. The SEA equations were solved one-hundred times with damping loss factors varying from 0.004 to 0.062 . Since ensemble variation was neglected for this analysis, the conditional density functions $f(y \mid x)$ were represented with Dirac delta functions. The predicted response levels were then computed using Eq. 4. The predicted responses for panels B and C are shown with the blue lines in Fig. 6. Notice that the spread in the predicted response is much larger when damping uncertainty is included in the analysis. It is also interesting to note that the solid blue lines are different than the solid black lines. Therefore the mean value of the response changes, by as 
much as $2.5 \mathrm{~dB}$ on panel $\mathrm{C}$, when damping uncertainty is included. As a result, it is important to account for damping uncertainty within SEA and not just use the mean damping loss factor for the analysis.

Finally the statistical variation in the loss factor was combined with the ensemble variation. Once again the SEA equations were solved one-hundred times with damping loss factors varying from 0.004 to 0.062 . This time the variance package in VA One was used to predict the ensemble variation for each run. The $90 \%$ confidence intervals predicted by VA One were then used to estimate conditional probability distributions $f(y \mid x)$. Figure 7 shows the conditional density functions for three different values of damping $x$. The three damping values represent the minimum, mean, and maximum damping loss factors considered in this analysis. Notice that as the sidewall damping increases the peaks shift to the left and become narrower. This indicates that both the velocity and the spread in the data are reduced as damping increases.

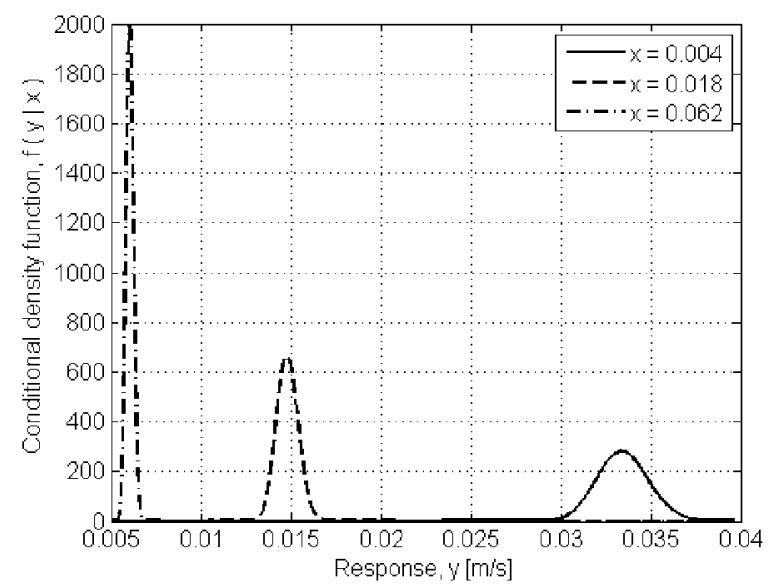

Fig. 7 - Conditional density functions for three different damping loss factors $(0.004,0.018$, and 0.062) at $2500 \mathrm{~Hz}$ in panel $B$.

The total response was found by combining the statistics of the damping loss factor, characterized by $g(x)$, with the conditional values $f(y \mid x)$ using Eq. 4 . Figure 8 shows the total, or unconditional, probability density function of the response, $h(y)$. This PDF accounts for both the statistical variation in the damping loss factor and the ensemble variation. The dashed red lines show the $90 \%$ confidence interval, which was determined by integrating under the curve and selecting the bounds such that $5 \%$ of the responses were greater than the upper bound and $5 \%$ were less than the lower bound. 


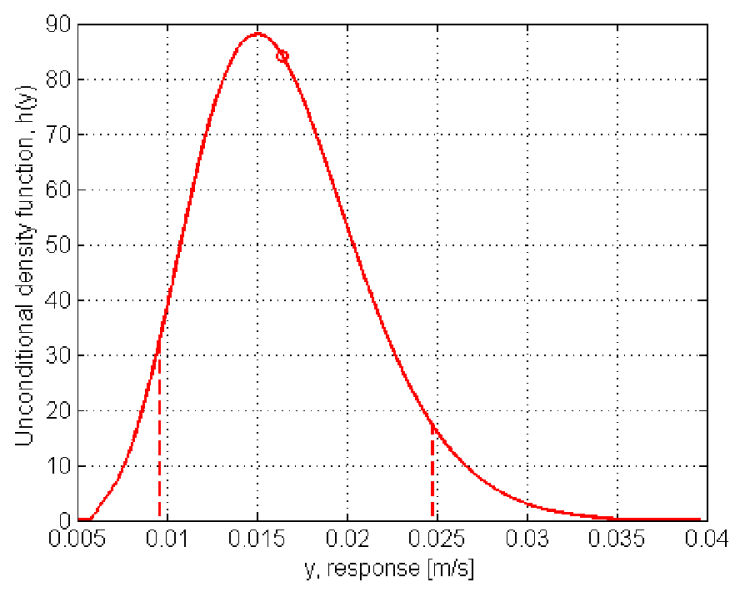

Fig. 8 - Unconditional density function for the $2500 \mathrm{~Hz} 1 / 3$-octave band, panel B.

The combined predictions accounting for both damping and ensemble variation are shown with the red lines in Fig. 6 . Since the red curves fell almost directly on top of the blue lines, they were shifted up by $1 / 3 \mathrm{~dB}$ for clarity. After comparing the blue and red curves, it is clear that ensemble variation has little impact on the predicted response. Therefore for this analysis, ensemble uncertainty could be neglected without a significant effect on the results.

\section{CONCLUDING REMARKS}

This paper demonstrates the need to account for measurement uncertainty within SEA. The analysis was performed on a stiffened, floor-equipped, composite cylinder. The impulse response decay method was used to estimate the damping of the sidewall from measured data. Damping uncertainty was then propagated through SEA. It was found that uncertainty in the damping loss factor has a much larger effect on the predicted response than ensemble variation. The results also demonstrate that damping uncertainty can have an impact on both the mean and variance of the predicted response. This has important implications. If SEA is performed using only mean damping loss factors then the predicted response may be inaccurate.

\section{APPENDIX A: SEA CYLINDER MODEL}

The SEA model of the cylinder is depicted in Fig. 9. The primary components of the model are the sidewall, stiffeners, floor, end caps, and acoustic cavities. The sidewall of the cylinder is modeled with singly curved shells consisting of 9 layers of $0.0001891 \mathrm{~m}$ thick T500-tape in a $\pm 45, \pm 32,90, \mp 32, \mp 45$ layup. The material properties for the tape are shown in Table 1 . In this case, the 1-and 2-directions lie in the plane of the material, with the 1-direction in line with the fiber orientation. 


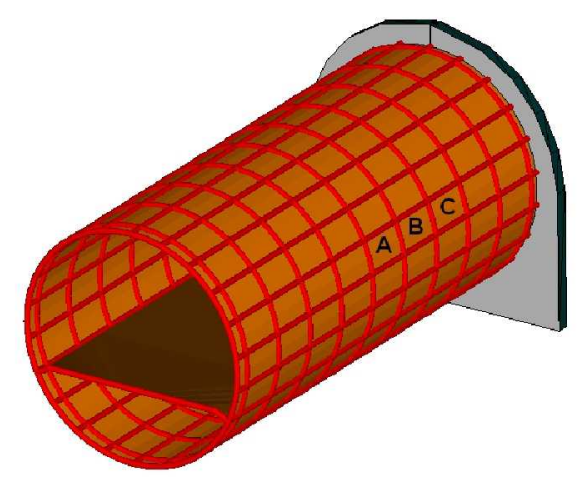

Fig. 9 - SEA model of the stiffened composite cylinder without the front end cap or acoustic cavities.

Table 1 - Material properties for T500-tape.

\begin{tabular}{|c|c|c|c|c|c|c|c|c|c|}
\hline Density $\left[\mathrm{kg} / \mathrm{m}^{3}\right]$ & \multicolumn{3}{|c|}{ Elastic Moduli $[\mathrm{Pa}]$} & \multicolumn{3}{c|}{ Poisson Ratios } & \multicolumn{3}{c|}{ Shear Moduli [Pa] } \\
\hline$\rho$ & $E_{1}$ & $E_{2}$ & $E_{3}$ & $v_{12}$ & $v_{23}$ & $v_{31}$ & $G_{12}$ & $G_{23}$ & $G_{31}$ \\
\hline 1590 & $1.51 \mathrm{e} 11$ & $8.96 \mathrm{e} 9$ & $8.96 \mathrm{e} 9$ & 0.3 & 0.15 & 0.15 & $5.14 \mathrm{e} 9$ & $5.14 \mathrm{e} 9$ & $5.14 \mathrm{e} 9$ \\
\hline
\end{tabular}

As depicted in Fig. 9, the sidewall is segmented into rectangular panels by frames in the circumferential direction and stringers in the axial direction. These stiffeners are modeled using the beams described in Table 2. Although the composite layup was different for the base, web, and crown (top) of each stiffener, the beam section definition in VA One only supports isotropic materials. Therefore the equivalent properties of the crown were used for the entire stiffener. The equivalent isotropic material properties for each type of stiffener are shown in Table 3 . Since the modal content of the stiffeners is low throughout the frequency range, they make poor SEA subsystems $^{14}$. However it was still important to include the stiffeners in the model to account for the impedance mismatch between the panels. The energy storage and dissipation within the beams was not modeled though.

Table 2 - Physical properties of the stiffeners.

\begin{tabular}{|c|c|c|c|c|c|c|c|c|}
\hline Beam & \multicolumn{4}{|c|}{ Moments } & \multicolumn{2}{c|}{ Cross Section } & \multicolumn{2}{c|}{ Shear Center Offset } \\
\hline & $I x x\left[\mathrm{~m}^{4}\right]$ & Iyy $\left[\mathrm{m}^{4}\right]$ & $J z z\left[\mathrm{~m}^{4}\right]$ & $Q z z\left[\mathrm{~m}^{4}\right]$ & $A\left[\mathrm{~m}^{2}\right]$ & $P[\mathrm{~m}]$ & $D x[\mathrm{~m}]$ & $D y[\mathrm{~m}]$ \\
\hline Stringer & $4.54 \mathrm{e}-9$ & $2.20 \mathrm{e}-8$ & $2.65 \mathrm{e}-8$ & $2.05 \mathrm{e}-10$ & $1.37 \mathrm{e}-4$ & 0.14 & 0 & $8.10 \mathrm{e}-3$ \\
\hline Frame & $7.60 \mathrm{e}-8$ & $1.15 \mathrm{e}-8$ & $8.75 \mathrm{e}-8$ & $2.01 \mathrm{e}-10$ & $1.82 \mathrm{e}-4$ & 0.21 & $-8.77 \mathrm{e}-3$ & $-6.95 \mathrm{e}-3$ \\
\hline
\end{tabular}

Table 3 - Isotropic material properties.

\begin{tabular}{|c|c|c|c|c|}
\hline Material & Density $\left[\mathrm{kg} / \mathrm{m}^{3}\right]$ & $\begin{array}{c}\text { Tensile Modulus } \\
{[\mathrm{Pa}]}\end{array}$ & $\begin{array}{c}\text { Shear Modulus } \\
{[\mathrm{Pa}]}\end{array}$ & Poisson Ratio \\
\hline Composite stringer & 1590 & $6.03 \mathrm{e} 10$ & $2.01 \mathrm{e} 10$ & 0.49 \\
\hline Composite frame & 1590 & $4.88 \mathrm{e} 10$ & $2.22 \mathrm{e} 10$ & 0.099 \\
\hline Plywood & 711 & $1.24 \mathrm{e} 10$ & $4.66 \mathrm{e} 9$ & 0.33 \\
\hline MDF & 730 & $2.80 \mathrm{e} 9$ & $1.04 \mathrm{e} 9$ & 0.35 \\
\hline
\end{tabular}


The $0.0127 \mathrm{~m}$-thick plywood floor and the $0.0889 \mathrm{~m}$-thick end caps are modeled as SEA plate subsystems with a damping loss factor of 0.03 . Meanwhile, the acoustic cavities were assumed to have a loss factor of 0.01 .

\section{REFERENCES}

1. H. Himelblau, D.L. Kern, F.E. Manning, A.G. Piersol, and S. Rubin, Dynamic environmental criteria: $N A S A-H D B K-7005$, NASA, (2001).

2. R.H. Lyon and R.G. DeJong, Theory and Application of Statistical Energy Analysis, Butterworth-Heinemann, Massachusetts, (1995).

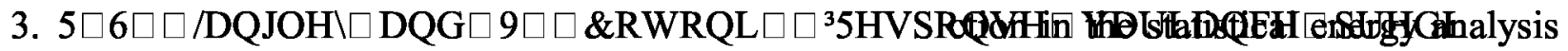
of built-XS $\square \mathrm{V} \backslash \mathrm{VWHP}$.VK'ooust. Soc. Am., 115(2), 706-718, (2004).

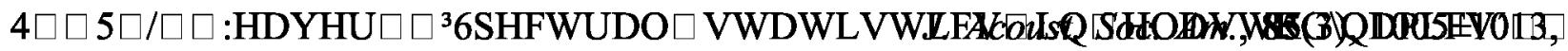
(1989).

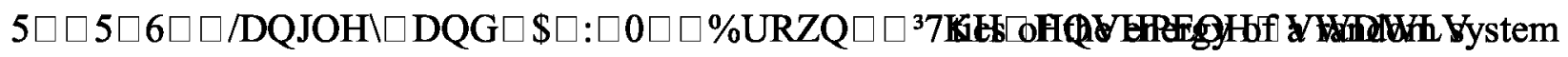

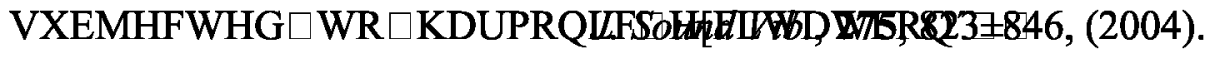

6. ESI Group, VA One 2008 User|s Guide, (2008).

7. 9 $\square \square$ \&RWRQL $\square \square$ 5 $\square$ 6 $\square \square /$ DQJOH $\square \square$ DQG $\square 0 \square 5 \square$ ) $\square \square$.LGQHU $\square \square^{3} 1$ XPHULFDO $\square$ DQG $\square$ H[SH variance prediction in the statistical energy analysis of built-XS $\square$ V $\backslash$ VWHP.VS\&und Vib., 288, 701-728, (2005).

8 $\square \square$ 5 $\square \square$ \&DEHOO $\square \square-\square \square$.ORV $\square \square$ 5 $\square \square \%$ XHKUOH $\square \square$ DQG $\square$ 1 $\square \square$ 6FKLOOHU $\square \square$ 39LEURDFRXV SDQHOV $\square \mathrm{DQG} \square \mathrm{F} \mid \mathrm{OL}$ (robtedaingss (2008).

9. \$ $\square$ \& $\square \square$-DFNVRQ $\square \square) \square-\square \square \%$ DOHQD $\square \square: \square / \square \square / \mathrm{D} \%$ DUJH $\square \square * \square \square$ 3HL $\square \square: \square \$ \square \square$ 3LWPDQ $\square \square$ D( composite fuselage technology - ,PSDFW $\square$ GIQDPLFV $\square$ DQG $\square$ DFRXVWLF $\square$ NWSSADQVPLVVLR Contractor Report 4035, (1986).

10. R. Cabell, N. Schiller, A. Allen, and M. Moeller, ${ }^{3 / R V V} \square$ IDFWRU $\square$ HVWLPDWLRQ $\square$ XVLQJ $\square$ WKH

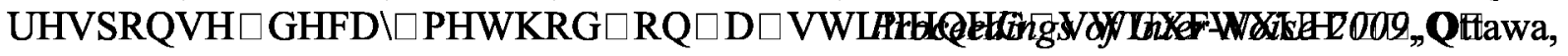
Canada, August 23-26, (2009).

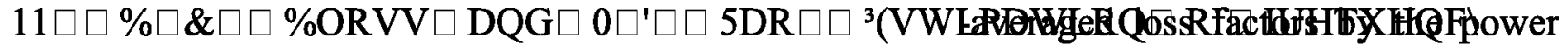
LQMHFWLRQ $\square$ DQG $\square$ WKH $\square$ LPSXOVH $\square$ UHVSKQACbtSLGSHEDXwP,HMK(R)G $\$ 40 \pm 249$, (2005).

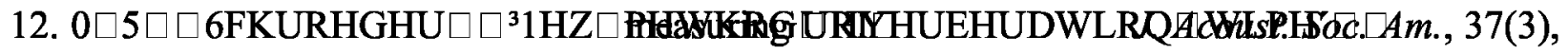
409-412, (1965). 
13. R.E. Walpole and R.H. Myers, Probability and statistics for engineers and scientists, Macmillan Publishing Company, New York, (1985).

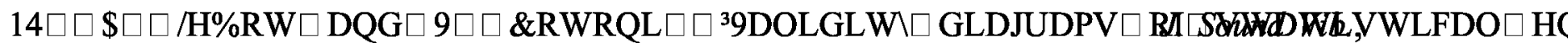
329, 221 \pm 235 , (2010). 